\title{
Hybrid Support Vector Regression and Genetic Algorithm Model for Tuning Magnetic Ordering Temperature of Manganite Refrigerant
}

\author{
${ }^{1}$ Abdullah Alqahtani, ${ }^{2,}{ }^{3}$ Taoreed O. Owolabi, ${ }^{4}$ Kabiru O. Akanded, \\ ${ }^{1}$ Sunday O. Olatunji and ${ }^{1}$ Nahier Aldhafferi \\ ${ }^{1}$ Department of Computer Information Systems, Imam Abdulrahman Bin Faisal University, \\ Dammam, Kingdom of Saudi Arabia \\ ${ }^{2}$ Department of Physics and Electronics, Adekunle Ajasin University, \\ Akungba Akoko, Ondo State, Nigeria \\ ${ }^{3}$ Department of Physics, King Fahd University of Petroleum and Minerals, \\ Dhahran, Kingdom of Saudi Arabia \\ ${ }^{4}$ School of Engineering, Institute for Digital Communications, \\ University of Edinburgh, Edinburgh, United Kingdom
}

\begin{abstract}
Manganite based materials have several unique properties which include low cost, environmental friendliness and huge magnetocaloric effect which make them suitable as magnetic refrigerant in Magnetic Refrigeration Ttechnology (MRT). However, effective utilization and deployment of this technology requires manganite refrigerants with magnetic ordering Temperature $\left(\mathrm{T}_{\mathrm{C}}\right)$ around ambient temperature. In order to tune the $T_{C}$ of manganite based materials to the desired ambient value and circumvent experimental stress therein, a hybrid of Support Vector Regression (SVR) Algorithm and Genetic Algorithm (GA) is proposed using the crystal lattice structural parameter of manganite based materials as the descriptors to the models. The generalization and predictive strength of the proposed hybrid GA-SVR Model is compared with the existing Gravitational Search Algorithm based model (GSA-SVR) on the basis of error reduction as well as computational complexity. The proposed hybrid GA-SVR Model outperforms the existing GSA-SVR Model with $8 \%$ and $2 \%$ performance improvement on the basis of the coefficient of correlation and root mean square error, respectively. Comparison of the proposed hybrid GA-SVR Model with the existing Manual Search based Support Vector Regression (MS-SVR) shows that the developed hybrid GA-SVR model outperforms MS-SVR Model with percentage improvement of $4.7 \%$ on the basis of correlation coefficient. Better performance demonstrated by GA-SVR Model coupled with its less computational time, strengthens its potential in enhancing room temperature magnetic refrigeration and promoting environmental friendly technology over ozone-depleting refrigeration technology.
\end{abstract}

Key words: Manganite based material, support vector regression, Genetic Algorithm, crystal lattice parameters and magnetic ordering temperature, technology, ozone

\section{INTRODUCTION}

The significance of refrigeration in everyday life cannot be overemphasized. Although, the conventional gas compression system of refrigeration is well-established and has enjoyed a great success but its environmental hazard such as the release of air pollutants and depletion of ozone layer pose a serious concern that needs urgent attention. Besides, the low efficiency of conventional gas-compression refrigeration reduces its effectiveness and makes it imperative to further research into alternative superior system of refrigeration. Magnetic
Refrigeration Technology (MRT) represents a potential substitute to the conventional system of refrigeration due to its utilization of non-hazardous refrigerant as well as its high efficiency (Khlifa et al., 2015). In addition, the low cost, absence of noise pollution and portability achieved in MRT make it a better alternative to the conventional gas compression system of refrigeration (Bruck, 2005; Gschneidner et al., 2005). These numerous advantages exhibited by MRT make it a good candidate for several applications such as large scale air conditioning, liquor distilling, heat pumps, large store refrigeration, gas liquefaction and waste separation among others

Corresponding Author: Owolabi Taoreed Olakunle, Department of Physics, King Fahd University of Petroleum and Minerals, Dhahran, Kingdom of Saudi Arabia 
(Phan and $\mathrm{Yu}, 2007$ ). The main challenge of MRT centers on the choice of manganite refrigerant with ambient magnetic ordering Temperature $\left(\mathrm{T}_{\mathrm{C}}\right)$ which is the temperature at which magnetic ordering of the material is destroyed due to thermal energy (Ghodhbane et al., 2014). This present research hybridizes Support Vector Regression (SVR) with Genetic Algorithm (GA) for developing robust model that estimates $T_{C}$ of manganite based materials subjected to doping using crystal lattice structural parameters as the descriptors to the model.

Support vector regression is a class of computational intelligent tools that has enjoyed a wide range of practical applications due to its unique features such as non-convergence to local minimal and minimization of generalized error bound (Cai et al., 2013; Owolabi et al., 2016a; Adewumi et al., 2016). The prediction and generalization capacity of SVR depends greatly on the proper selection of its parameters which include the regularization factor $(C)$, epsilon $(\varepsilon)$, kernel function, kernel option $(\eta)$ and hyper-parameter lambda $(\lambda)$ (Owolabi et al., 2016b-f). In order to develop SVR-based Model with high degree of accuracy and excellent generalization ability for estimating magnetic ordering temperature of manganite refrigerant without the conventional experimental challenges, SVR is hybridized with GA to develop GA-SVR Model. The uniqueness of GA over other Heuristic Algorithms include its ability to code parameters set (strings) instead of parameters themselves and the use of probabilistic and non-deterministic rules (Hosseini et al., 2016). These unique properties coupled with the novelty of SVR are employed in this present research to tune the $T_{C}$ of manganite based materials towards ambient condition for MRT application.

The working principle of MRT utilizes magnetocaloric effect which measures the response of magnetic material to the applied external magnetic field. Alignment of electronic spins of manganite along the axis of applied field leads to a reduction in the entropy of the system. In an attempt to compensate for this response, atoms of the material begins to vibrate which consequently raises the thermal energy as well as the temperature of the manganite (Wang and Jiang, 2013). If the $\mathrm{T}_{\mathrm{C}}$ of the manganite is around room temperature, the thermal energy can easily results into magnetic transition; otherwise, the MRT becomes inefficient. The electronics spins resume their random orientation after the removal of the field which lowers the rate of vibration of atoms due to increase in entropy of the system. This action lowers the temperature of the system. The change in temperature of the system due to the variation of the applied magnetic field (phenomenon known as magnetocaloric effect) results into magnetic refrigeration. Efficient MRT deserves large magnetocaloric effect which is controlled by the magnetic ordering temperature, since, this effect attains its maximum value at the $\mathrm{T}_{\mathrm{C}}$ of manganite. Large magnetocaloric effect of manganite based material as well as the possibility of altering its $\mathrm{T}_{\mathrm{C}}$ to a desired value near room temperature through doping makes it unique among other magnetic refrigerants.

The results of the hybrid GA-SVR Model show that the developed model is capable of estimating the $T_{C}$ of doped manganite based materials with high degree of accuracy. Implementation of the proposed hybrid model would definitely enhance tuning of magnetic ordering temperature of manganite-based materials to the desired application level and significantly reduces the dependence on ozone-depleting refrigeration technology which is harmful to the entire globe.

Background of the proposed hybrid model: This study presents the description of support vector regression algorithm and genetic algorithm.

Description of the proposed support vector regression: Support vector regression is an extension of support vector machines that was originally developed for classification problems (Cortes and Vapnik, 1995). Its formulation is basically structured using statistical learning theory and structural risk minimization principle (Basak et al., 2007; Vapnik, 2013). The inductive principle of structural risk minimization entails simultaneous minimization of Vapnik-Chervonenkis dimension as well as the associated empirical risk. This gives SVR a unique feature of error bound generalization which measures the training error and a regularization term that influences the hypothesis space complexity. SVR constructs hyper-plane in high dimensional feature space and the distance of the hyperplane to the nearest data-points is controlled by the loss function (Thenmozhi and Chand, 2016; Owolabi et al., 2016b-f). The loss function penalizes the estimation error greater than the specified threshold. The threshold value of error termed epsilon determines the number of support vectors utilized in constructing the regression function. Training in SVR is tantamount to providing solution to a linearly constrained quadratic programing problem and this unique feature enhances SVR solution to attain the global minimal unlike other computational intelligence tools that can be easily trapped in local minima due to non-linear optimization. Equation 1 presents the formulation of SVR in which $\left(\Phi_{0}\right)=1$ so, that $\left(\omega_{0}\right)$ equals the bias term that can be dropped by preprocessing the data to zero mean: 


$$
\mathrm{F}(\mathrm{x}, \omega)=\sum_{\mathrm{i}=0}^{\mathrm{N}} \omega_{1} \Phi_{\mathrm{i}}=\omega^{\mathrm{T}} \Phi(\mathrm{x})
$$

where: $\omega=\left(\omega_{0}, \omega_{1}, . ., \omega_{\mathrm{n}}\right)^{\mathrm{T}}=$ represents the weight $\Phi$ $\left.(\mathrm{x})=\Phi_{0}(0), \Phi_{1}(\mathrm{x}), \ldots, \Phi_{\mathrm{i}}(\mathrm{x})\right)=$ contains the input lattice parameters.

Equation 2 represents the empirical risk as described by Thenmozhi and Chand (2016) and Adewumi et al. (2016):

$$
\mathrm{r}_{\mathrm{e}}=\frac{1}{\mathrm{~N}} \sum_{\mathrm{i}=1}^{\mathrm{N}} \mathrm{L}\left(\mathrm{T}_{\mathrm{C}_{\mathrm{i}}}, \mathrm{F}(\mathrm{x}, \omega)\right)
$$

where, the $\varepsilon$ loss function $\mathrm{L}\left(\mathrm{T}_{\mathrm{c}}, \mathrm{F}(\mathrm{x}, \mathrm{w})\right)$ is defined as:

$$
L\left(T_{C}, F(x, \omega)\right)=\left\{\begin{array}{cc}
\left|T_{C}, F(x, \omega)\right|-\varepsilon, & \left|T_{C}, F(x, \omega)\right| \geq \varepsilon \\
0 & \text { otherwise }
\end{array}\right.
$$

With the introduction of non-negative slack variables $\left(\xi_{\mathrm{i}}\right.$ and $\left.\xi^{*}\right)$, the optimization problem can be modified by minimization of Eq. 4 subjected to constraints contained in Eq. 5:

$$
\begin{gathered}
\Phi\left(\omega, \xi_{i}, \xi_{i}^{*}\right)=C\left(\sum_{i=1}^{N}\left(\xi_{i}+\xi_{i}^{*}\right)+\frac{1}{2} \omega^{\mathrm{T}} \omega\right. \\
\left\{\begin{array}{c}
\mathrm{T}_{\mathrm{C}_{\mathrm{i}}}-\omega^{\mathrm{T}} \Phi\left(\mathrm{x}_{\mathrm{i}}\right) \leq \varepsilon+\xi_{\mathrm{i}} \\
\omega^{\mathrm{T}} \Phi\left(\mathrm{x}_{\mathrm{i}}\right)-\mathrm{T}_{\mathrm{C}_{\mathrm{i}}} \leq \varepsilon+\xi_{\mathrm{i}}^{*} \\
\xi_{\mathrm{i}} \geq 0 \\
\xi_{\mathrm{i}}^{*} \geq 0, \mathrm{i}=1,2, \ldots, \mathrm{N}
\end{array}\right.
\end{gathered}
$$

where, $\mathrm{C}$ is the regularization factor that controls the dimension of Vapnik-Chervonenkis.

By solving the optimization problem using lagrange multipliers $\left(\alpha_{i}, \alpha^{*}\right)$, the decision function is presented in Eq. 6 with inclusion of non-linear mapping function (Kernel function) described by Mercer's Theorem presented in Eq. 7:

$$
\begin{gathered}
\mathrm{F}(\mathrm{x}, \omega)=\sum_{\mathrm{i}=1}^{\mathrm{N}}\left(\alpha_{\mathrm{i}}-\alpha_{\mathrm{i}}^{*}\right) \mathrm{K}\left(\mathrm{x}_{\mathrm{i}}, \mathrm{x}\right) \\
\mathrm{K}\left(\mathrm{x}_{\mathrm{i}}, \mathrm{x}\right)=\Phi^{\mathrm{T}}\left(\mathrm{x}_{\mathrm{i}}\right) \Phi(\mathrm{x})
\end{gathered}
$$

The actual Kernel function utilized in this present research is radial basis function (Gaussian kernel function) presented in Eq. 8:

$$
\mathrm{K}\left(\mathrm{x}_{\mathrm{i}}, \mathrm{x}\right)=\exp \left(-\gamma\left\|\overrightarrow{\mathrm{x}_{\mathrm{i}}}-\overrightarrow{\mathrm{x}}\right\|^{d}\right)
$$

where, $\gamma$ and $d$ are Kernel parameters SVR hyper-parameters such as the regularization factor $\mathrm{C}, \varepsilon$ and kernel option determine the generalization and predictive strength of SVR-based model (Owolabi et al., $2016 \mathrm{a}-\mathrm{c}$ ). In order to develop robust model that ensures global minima, these parameters are tuned using GA Heuristic Algorithm. The hybrid GA-SVR Model captures the magnetic ordering temperature of manganite based materials accurately and its implementation will be of immense significance in ensuring efficient pollutant free system of refrigeration.

Description of Genetic Algorithm (GA): GA is a class of heuristic algorithm that is based on the principle of natural selection and genetics. It was developed around 1970 , by John Holland and his collaborators. Operations in GA include selective reproduction, crossover and mutation. It generates a set of feasible initial solutions referred to as initial population and individuals in the population are named as chromosomes. Chromosomes of a population are potential solutions of the problem and are characterized by genes (characters) (Liang et al., 2013; Wen et al., 2016). The quality or strength of each of the chromosome of the population is measured by the defined fitness function. The parents are selected purposely for reproduction coupled with a crossover operator and the corresponding offspring chromosomes are generated. Crossover probability plays a significant rule in the reproduction process by ensuring that the generated offspring inherit varying characters from the parents. Changing the offspring chromosomes locally would ultimately results into random mutation operation which gives room for transference of the best chromosomes to the new generation through a defined elitism mechanism. The chromosomes in the new generation are evaluated using fitness function and the process continues until the defined termination condition is reached. Mutation is a very vital process in GA, since, it circumvents local minimum (Hosseini et al., 2016). GA quickly converges to local minimum if the operators were constrained within selection and crossover. This is due to the fact that the algorithm quickly eliminates individuals with poor measure of performance until individuals in the population are typically identical. Hence, the solution comes to abrupt stop without the new influx of genetic materials. In order to avert this premature convergence and ultimately ensure global minimum, some genes are subjected to the process of mutation. Through repetition of selection, crossover and mutation for many generations, global optimum could be guaranteed. 
Res. J. Applied Sci., 13 (1): 87-93, 2018

\section{MATERIALS AND METHODS}

Empirical study: This study describes the computational hybridization of the two algorithms (GA and SVR). The description of the data set used for modeling and simulation is also presented.

Description of data set: This present research utilizes 75 experimental crystal lattice structural parameters and their corresponding magnetic ordering temperature for developing and validating the proposed models. The experimental data was drawn from the literature (Wang and Jiang, 2013; Selmi et al., 2015a-c; Mahjoub et al., 2014; $\mathrm{Ca}$ and $\mathrm{Fe}, 2014$; Mleiki et al., 2015.

Table 1 presents the statistical analysis of the dataset and useful insight regarding the range and the content of the data-points can be easily deduced from the value of mean, maximum as well as the minimum. The intricacies and disparities in the data-points can as well be inferred from the presented standard deviations. Correlation coefficients between each pair of the crystal lattice parameter (descriptor) and the magnetic ordering temperature (target) are also presented in the table. Correlation coefficient measures the degree of linear relationship between the descriptor and target and the values obtained from the analysis show that linear model cannot adequately capture the relationship between the lattice distortion and magnetic transition temperature observed experimentally. Therefore, the need for nonlinear model with generalized error bound is highly essential for ensuring robust model. The choice of SVR as the computational intelligence tool is due to its unique features in maintaining high generalization and predictive ability in the presence of few data-points, since, few data-points characterizes this field (Owolabi et al., 2016a).

Computational hybridization of GA with SVR: SVR hyper-parameters were optimized using GA within MATLAB computing environment. Before the optimization procedures, the dataset which comprises of experimental crystal structural lattice parameters of several manganite based materials and their corresponding magnetic ordering temperature was subjected to randomization so as to ensure efficient and unbiased partitioning of dataset into training and testing set. The training dataset consists of sixty data-points $(80 \%$ of the total dataset) while the testing dataset consists of fifteen data-points ( $20 \%$ of the total dataset). The computational descriptions of the hybridization are summarized:

Step 1: Randomization and separation of dataset (seventy five experimental lattice parameters and their corresponding magnetic ordering temperature) into training and testing set in the ratio of eight to two.

\begin{tabular}{lccrc}
\multicolumn{5}{l}{ Table 1: Statistical analysis of the data-set } \\
\hline Factors & $\mathrm{a}(\AA)$ & $\mathrm{b}(\AA)$ & $\mathrm{c}(\AA)$ & \multicolumn{1}{c}{$\mathrm{T}_{\mathrm{C}}(\mathrm{K})$} \\
\hline Mean & 5.482 & 6.570 & 7.943 & 235.55 \\
SD & 0.032 & 1.255 & 3.317 & 95.92 \\
Maximum & 5.543 & 7.760 & 13.597 & 370.000 \\
Minimum & 5.429 & 5.438 & 5.419 & 18.520 \\
Correlation coefficient (\%) & 53.180 & -1.300 & 19.300 & \\
\hline
\end{tabular}

Step 2: Generation of initial population of SVR hyper-parameter using GA.

Step 3: Training of SVR Algorithm with training dataset using all hyper-parameters generated in Step 2.

Step 4: Validate the trained SVR models using testing dataset and calculate RMSE.

Step 5: Based on Step 5, generate mating pool, select parents, apply crossover and mutation operators to generate new population of hyper-parameters.

Step 6: Repeat Step 3-5 until converged minimum RMSE is obtained and the algorithm is terminated. The optimum hyper-parameters and the measures of estimation accuracy are saved.

\section{RESULTS AND DISCUSSION}

This study presents and discusses the results of the modeling and simulation. The results of the developed GA-SVR Model are also compared with the existing models (MS-SVR and GSA-SVR) for magnetic ordering temperature estimation

Investigating the effect of number of population on the performance of GA-SVR Model: The influence of the number of population on the performance of GA-SVR Model was investigated and presented in Fig. 1. The Figure 1 shows different convergence when different numbers of population were used $100,0.8,0.65$ and 0.009 number of generation, selection probability, cross-over probability and mutation probability, respectively. The results converge to lower RMSE value as the number of population increases up to a point beyond which increase in the number of population has little or no significance on the model performance. Since, small number of initial population limits the chance of global solution to emerge from the onset and promotes local minimum convergence, this can be observed in Fig. 1 as the solutions converge to local minimum at initial population of 10 and 20 . The model becomes more robust at high number of population as depicted in the graph. The corresponding computational time complexity is depicted in Fig. 2 . The time complexity of the model increases with increase in the number of 


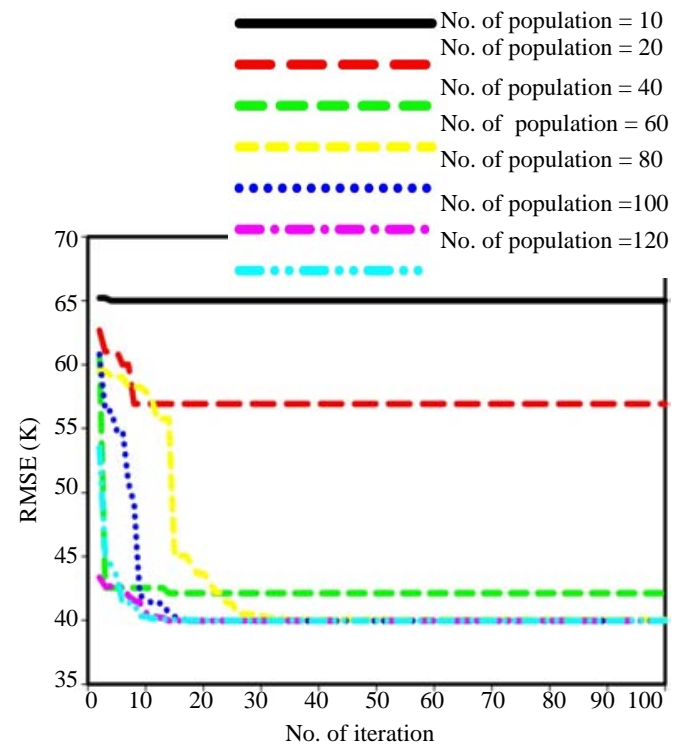

Fig. 1: Sensitivity of the GA-SVR Model to number of population

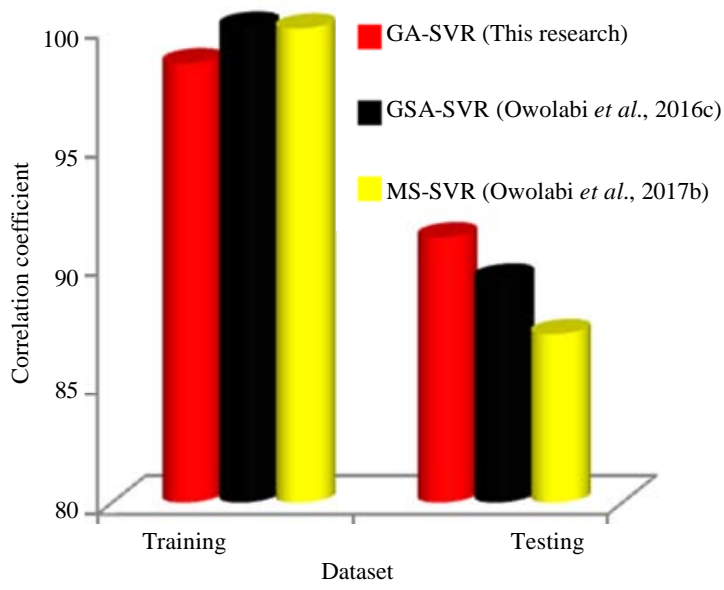

Fig. 2: Comparison of the generalization and predictive capacity of the developed hybrid GA-SVR Model with the existing models

population. In order to strike a balance between time complexity and the performance of the model, the optimum number of population was set at 60 . The optimum values of SVR hyper-parameters obtained using GA are presented in Table 2 .

Comparison of the performance of the developed hybrid GA-SVR Model with existing models: The generalization and predictive ability of the developed hybrid GA-SVR Model is compared with the existing GSA-SVR (Owolabi et al., 2016) Model and MS-SVR (Owolabi et al., 2017) Model on the basis of the Correlation Coefficient
Table 2: Optimum hyper-parameters of the developed hybrid GA-SVR Model

\begin{tabular}{ll}
\hline SVR hyper-parameter & Optimum value from GA \\
\hline Regularization factor & 93.0956 \\
Lambda & E-7 \\
Epsilon & 0.1087 \\
Kernel option & 0.0089 \\
Kernel function & Gaussian function \\
\hline
\end{tabular}

(CC ) between the estimated and experimental magnetic ordering temperature for both training and testing set of data. Figure 2 shows the comparison.

Capacity of the developed hybrid GA-SVR Model with the existing models: The present hybrid GA-SVR Model outperforms other existing models such as GSA-SVR and MS-SVR with 1.9 and $4.7 \%$ improvement, respectively. This improvement would definitely improve the performance of magnetic refrigeration when implemented.

\section{CONCLUSION}

Hybrid GA-SVR Model is developed for estimating magnetic ordering temperature of manganite based materials. The generalization and predictive capacity of the developed hybrid model outperforms other existing models such as GSA-SVR and MS-SVR Model with performance improvement of 1.9 and $4.7 \%$, respectively.

\section{IMPLEMENTATIONS}

Implementation of the proposed hybrid model would definitely enhance tuning of magnetic ordering temperature of manganite-based materials to the desired application level and significantly reduces the dependence on ozone-depleting refrigeration technology which is harmful to the entire globe.

\section{ACKNOWLEDGEMENT}

Support from university of Dammam is acknowledged.

\section{REFERENCES}

Adewumi, A.A., T.O. Owolabi, I.O. Alade and S.O. Olatunji, 2016. Estimation of physical, mechanical and hydrological properties of permeable concrete using computational intelligence approach. Appl. Soft Comput., 42: 342-350.

Basak, D., S. Pal and D.C. Partababis, 2007. Support vector regression. Neural Inf. Process. Lett. Rev., 11: 203-224. 
Bruck, E., 2005. Developments in magnetocaloric refrigeration. J. Phys. D. Appl. Phys., 38: R381-R391.

$\mathrm{Ca}, \mathrm{B}$. and M. Fe, 2014. A large magnetic entropy change near room temperature in La0.8Ba0.1 Ca0.1 Mn0.97Fe0.0303 perovskite. J. Alloys Compd., 600: 172-177.

Cai, C.Z., T.T. Xiao, J.L. Tang and S.J. Huang, 2013. Analysis of process parameters in the laser deposition of $\mathrm{Yba} 2 \mathrm{Cu} 3 \mathrm{O} 7$ superconducting films by using SVR. Phys. C. Supercond., 493: $100-103$.

Cortes, C. and V. Vapnik, 1995. Support-vector networks. Mach. Learn., 20: 273-297.

Ghodhbane, S., E. Tka, J. Dhahri and E.K. Hlil, 2014. A large magnetic entropy change near room temperature in $\mathrm{La} 0.8 \mathrm{Ba0} .1 \mathrm{Ca} 0.1 \mathrm{Mn} 0.97 \mathrm{Fe} 0.03 \mathrm{O} 3$ perovskite. J. Alloys Compd., 600: 172-177.

Gschneidner Jr, K.A., V.K. Pecharsky and A.O. Tsokol, 2005. Recent developments in magnetocaloric materials. Rep. Prog. Phys., 68: 1479-1539.

Hosseini, M., S.A.M. Naeini, A.A. Dehghani and Y. Khaledian, 2016. Estimation of soil mechanical resistance parameter by using particle swarm optimization, genetic algorithm and multiple regression methods. Soil Tillage Res., 157: 32-42.

Khlifa, H.B., Y. Regaieg, W. Cheikhrouhou-Koubaa, M. Koubaa and A. Cheikhrouhou, 2015. Structural, magnetic and magnetocaloric properties of $\mathrm{K}$-doped Pr0.8Na0.2-xKxMnO3 manganites. J. Alloys Compd., 650: 676-68.

Liang, G., J. Xu and L. Liu, 2013. QSPR analysis for melting point of fatty acids using genetic algorithm based multiple linear regression (GA-MLR). Fluid Phase Equilib., 353: 15-21.

Mahjoub, S., M. Baazaoui, R. M'nassri, H. Rahmouni and N.C. Boudjada et al., 2014. Effect of Iron substitution on the structural, magnetic and magnetocaloric properties of $\mathrm{Pr} 0.6 \mathrm{Ca} 0.1 \mathrm{Sr} 0.3 \mathrm{Mnl}-\mathrm{x}$ Fex O3 $(0=x=0.075)$ manganites. J. Alloys Compd., 608: 191-196.

Mleiki, A., S. Othmani, W. Cheikhrouhou-Koubaa, M. Koubaa and A. Cheikhrouhou et al., 2015. Effect of praseodymium doping on the structural, magnetic and

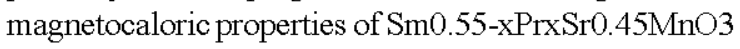
$(0.1=\mathrm{x}=0.4)$ manganites. J. Alloys Compd., 645: 559-565.

Owolabi, T.O., K.O. Akande and S.O. Olatunji, 2016a. Application of computational intelligence technique for estimating superconducting transition temperature of YBCO superconductors. Appl. Soft Comput., 43: 143-149.
Owolabi, T.O., K.O. Akande and S.O. Olatunji, $2016 \mathrm{~b}$. Computational intelligence approach for estimating superconducting transition temperature of disordered MgB2 superconductors using room temperature resistivity. Appl. Comput. Intell. Soft Comput., 2016: $1-7$

Owolabi, T.O., K.O. Akande and S.O. Olatunji, $2016 \mathrm{c}$. Computational intelligence method of estimating solid-liquid interfacial energy of materials at their melting temperatures. J. Intell. Fuzzy Syst., 31: 519-527.

Owolabi, T.O., K.O. Akande and S.O. Olatunji, 2017a. Estimation of average surface energies of transition metal nitrides using computational intelligence technique. Soft Comput, 21: 6175-6182.

Owolabi, T.O., K.O. Akande, S.O. Olatunji, A. Alqahtani and N. Aldhafferi, 2016d. Estimation of curie temperature of manganite-based materials for magnetic refrigeration application using hybrid gravitational based support vector regression. AIP. Adv., 6: 105009-1-105009-12.

Owolabi, T.O., K.O. Akande, S.O. Olatunji, A. Alqahtani and N. Aldhafferid, 2017b. Modeling of curie temperature of manganite for magnetic refrigeration application using manual search and hybrid gravitational-based support vector regression. Soft Comput., 6: 1-10.

Owolabi, T.O., M. Faiz, S.O. Olatunji and I.K. Popoola, 2016e. Computational intelligence method of determining the energy band gap of doped $\mathrm{ZnO}$ semiconductor. Mater. Des., 101: 277-284.

Owolabi, T.O., Y.F. Zakariya, S.O. Olatunji and K.O. Akande, 2016. Estimation of melting points of fatty acids using homogeneously hybridized support vector regression. Neural Comput. Appl., 1: 1-13.

Phan, M.H. and S.C. Yu, 2007. Review of the magnetocaloric effect in manganite materials. J. Magn. Magn. Mater., 308: 325-340.

Selmi, A., R. M'nassri, W. Cheikhrouhou-Koubaa, N.C. Boudjada and A. Cheikhrouhou, 2015a. Effects of partial Mn-substitution on magnetic and magnetocaloric propertiesin Pr0.7Ca0.3Mn0.95X0.0 $5 \mathrm{O} 3(\mathrm{Cr}, \mathrm{Ni}, \mathrm{Co}$ and $\mathrm{Fe}$ ) manganites. J. Alloys Compd., 619: 627-633.

Selmi, A., R. M'nassri, W. Cheikhrouhou-Koubaa, N.C. Boudjada and A. Cheikhrouhou, 2015b. Influence of transition metal doping ( $\mathrm{Fe}, \mathrm{Co}, \mathrm{Ni}$ and $\mathrm{Cr}$ ) on magnetic and magnetocaloric properties of Pr0.7Ca0.3MnO3 manganites. Ceram. Intl., 41: 10177-10184.

Selmi, A., R. M'nassri, W. Cheikhrouhou-Koubaa, N.C. Boudjada and A. Cheikhrouhou, 2015c. The effect of Co doping on the magnetic and magnetocaloric properties of $\mathrm{Pr} 0.7 \mathrm{CaO} .3 \mathrm{Mnl}-\mathrm{xCoxO} 3$ manganites. Ceram. Intl., 41: 7723-7728. 
Thenmozhi, M. and G.S. Chand, 2016. Forecasting stock returns based on information transmission across global markets using support vector machines. Neural Comput. Appl., 27: 805-824.

Vapnik, V.N., 2013. The Nature of Statistical Learning Theory. 2nd Edn., Springer, Berlin, Germany, ISBN:978-1-4757-3264-1, Pages: 313.
Wang, Z. and J. Jiang, 2013. Magnetic entropy change in perovskite manganites La 0.7A0.3 Mn O3 La0.7A0.3Mn0.9Cr0.1O3 $(\mathrm{A}=\mathrm{Sr}, \mathrm{Ba}, \mathrm{Pb})$ and Banerjee criteria on phase transition. Solid State Sci., 18: 36-41.

Wen, J., H. Yang, X. Tong, K. Li and S. Wang et al., 2016. Optimization investigatioon configuration parameters of serrated fin in plate-fin heat exchanger using genetic algorithm. Intl. J. Therm. Sci., 101: 116-125. 